\title{
PERCEPCIÓN DE UN GRUPO DE DOCENTES DE I Y II CICLO DE EDUCACIÓN GENERAL BÁSICA DE ESCUELAS PÚBLICAS DE HEREDIA SOBRE LOS TEMAS DE ESTADÍSTICA Y PROBABILIDAD PERCEPTIONS TOWARDS STATISTICS AND PROBABILITY OF PRIMARY SCHOOL TEACHERS FROM THE REGION OF HEREDIA
}

\section{Volumen 15, Número 1}

Enero - Abril

pp. 1-23

Este número se publicó el $1^{\circ}$ de enero de 2015

DOI: dx.doi.org/10.15517/aie.v15i1.17728

\author{
Marianela Alpízar Vargas \\ Laura Chavarría Oviedo \\ Katalina Oviedo Rodríguez
}

Revista indizada en REDALYC, $\underline{\text { SCIELO }}$

Revista distribuida en las bases de datos:

CATÁLOGO DE LATINDEX, IRESIE, CLASE, DIALNET, DOAJ, E-REVIST@S, SHERPA/ROMEO, QUALIS, MIAR

Revista registrada en los directorios:

ULRICH'S, REDIE, RINACE, OEI, MAESTROTECA, PREAL, CLACSO 


\title{
PERCEPCIÓN DE UN GRUPO DE DOCENTES DE I Y II CICLO DE EDUCACIÓN GENERAL BÁSICA DE ESCUELAS PÚBLICAS DE HEREDIA SOBRE LOS TEMAS DE ESTADÍSTICA Y PROBABILIDAD PERCEPTIONS TOWARDS STATISTICS AND PROBABILITY OF PRIMARY SCHOOL TEACHERS FROM THE REGION OF HEREDIA
}

\author{
Marianela Alpízar Vargas ${ }^{1}$ \\ Laura Chavarría Oviedo ${ }^{2}$ \\ Katalina Oviedo Rodríguez $z^{3}$
}

\begin{abstract}
Resumen: Los temas de estadística y probabilidad se encuentran presentes en los Programas de Estudio del Ministerio de Educación Pública de Costa Rica desde 1995; sin embargo la poca formación que han recibido los docentes de I y II Ciclo de la Educación General Básica en dichos temas. El presente artículo expone resultados de una investigación que se realizó, con 20 docentes, durante un proceso de capacitación sobre Didáctica de la Probabilidad y la Estadística en la Dirección Regional de Heredia en el 2011. La información se recolectó por medio de cuestionarios auto administrados. El objetivo fue describir la percepción que tiene este grupo de docentes de primaria en ejercicio de la Dirección Regional de Heredia ante el proceso de enseñanza y aprendizaje de los temas de estadística y probabilidad. Entre los resultados obtenidos, los docentes son conscientes de que los temas citados deben enseñarse desde los primeros niveles de formación, algunos les temen por su nivel de dificultad, otros no los imparten en sus clases porque no les da tiempo y otros desconfían de su fiabilidad. Una de las conclusiones del estudio es la necesidad que tienen los entes formadores de revisar y modificar sus planes de formación profesional, para adecuarlos a las exigencias actuales y brindar procesos de capacitación y actualización a docentes en ejercicio.
\end{abstract}

Palabras clave: ESTADÍSTICA, PROBABILIDAD, DOCENTES, EDUCACIÓN PRIMARIA, EDUCACIÓN CONTINUA, CURRICULUM, COSTA RICA

Abstract: In Costa Rica, the topics of statistics and probability have been included in the Basic General Education curricula of the Ministry of Public Education, since 1995. However, the poor academic formation that elementary school educators have received causes them to develop unfavorable attitudes towards these topics. This paper presents results of a study that was conducted with 20 teachers during a training process on Teaching Probability and Statistics in the Regional Directorate of Heredia in the 2011. Data was collected through questionnaires. The purpose of this article was to describe the perception that this group of in-service elementary school teachers, of the Regional Directorate of Heredia, has in relation to teaching and learning of topics of statistics and probability. Although these teachers are aware that statistics and probability must be taught since elementary school, some of them are afraid to face them and even have made the decision to leave them out of the curriculum motivated by their lack of confidence in their own capacity to teach such complex topics, others not taught them in their classes because lack of time or because of distrust in its reliability. One of the main conclusions of this study is that Universities should modify their curriculum to adapt it to the current requirements and also they should offer training processes, such as workshops, for teachers.

Key words: STATISTICS, PROBABILITY, PRIMARY EDUCATION, TEACHERS, CONTINUING EDUCATION, COSTA RICA

\footnotetext{
1 Académica, Escuela de Matemática, Universidad Nacional de

Costa Rica. Dirección electrónica: malpiza@una.cr

2 Docente, Colegio Nueva Esperanza, Costa Rica. Dirección electrónica: merula13986@hotmail.com

${ }^{3}$ Brasil. Dirección electrónica: kataor@hotmail.es
}

Artículo recibido: 23 de mayo, 2014

Enviado a corrección: 10 de octubre, 2014

Aprobado: 8 de diciembre, 2014 


\section{Introducción}

La estadística y la probabilidad como hoy se conocen, surgen luego de un largo proceso y como respuesta a diversas necesidades de los seres humanos. En un principio el objeto de estudio de la estadística fue generar información útil para los gobiernos como ayuda en su administración. Los censos de población para el control del pago de impuestos, así como el interés en el registro de estadísticas vitales como nacimientos, matrimonios y defunciones fueron fundamentales en esta primera etapa; luego, con el paso de los años, fue reconocida como disciplina para diversas actividades que se realizan, tanto en la ciencia como en situaciones que se presentan en la vida cotidiana.

La probabilidad también ha alcanzado mucho prestigio, su inicio estuvo muy vinculado con la solución de problemas relativos a juegos de azar, pero con el paso de los años fue incrementando su importancia en diferentes áreas, como las ciencias ambientales, sociales y médicas.

En la actualidad estas temáticas han logrado ganar espacios en muchos ámbitos, son indispensables en estudios de poblaciones y tienen muchas aplicaciones; su importancia ha estimulado que también los sectores no profesionales necesiten conocer sus principios esenciales. Por lo anterior, es fundamental que toda persona, posea conocimientos básicos en estos temas, razón por la cuál se dio su inclusión en los programas de estudio de la Educación General Básica del país.

Dada la incorporación de estas temáticas, en los programas de estudio, es esencial que los responsables de enseñarlas en los centros educativos estén conscientes del carácter instrumental que tienen en innumerables contextos y que posean un dominio de los conceptos básicos de las mismas; sin embargo, algunas investigaciones mencionan que los docentes no tienen formación en principios teóricos de dichos temas, en sus aplicaciones y en su enseñanza, tan poco tienen pleno conocimiento de las propuestas metodológicas planteadas por el MEP; la formación profesional no ha sido suficiente y los procesos de actualización y capacitación son prácticamente nulos; además, los docentes enfrentan limitaciones y dificultades, entre ellas: preparación recibida, tiempo limitado para enseñar los temas y falta de recursos adecuados (Alpízar, Barrantes, Bolaños, Céspedes, Delgado, Freer, Padilla y Víquez, 2012; Barrantes, Bolaños, Céspedes, Delgado, Freer, Padilla y Víquez, 2010; Chaves, 2007 y Jiménez y Jiménez 2005).

Muchos de los inconvenientes que presenta el proceso de enseñanza y aprendizaje de estadística y probabilidad, se dan porque los docentes no reconocen su utilidad en la vida 
cotidiana, por ello se estima que el cambio de la enseñanza dependerá del grado en que se pueda convencer a los docentes de que estas son temáticas útiles para los estudiantes (Gattuso, 2006).

Este artículo es parte de una investigación realizada durante el 2011 con 20 docentes de primaria de la Dirección Regional de Heredia que asistieron a un proceso de capacitación. Es un extracto del trabajo de graduación Actitudes y conocimientos que tienen docentes de la educación general básica costarricense de I y ll ciclo sobre estadística y probabilidad, en la Dirección Regional Educativa de Heredia, presentado como requisito para el grado de licenciatura en 2013 en la Universidad Nacional; además, esta investigación estuvo vinculada con los proyectos Didáctica de la probabilidad y la estadística en primaria y La utopía en la enseñanza de las Matemáticas en primaria ambos adscritos y financiados por la Escuela de Matemática de la Universidad Nacional de Costa Rica.

De manera específica, el objetivo de este artículo es describir la actitud que tuvo un grupo de docentes de primaria en ejercicio de la Dirección Regional de Heredia, sobre los temas de estadística y la probabilidad, durante un proceso de capacitación sobre didáctica en dichos temas.

\section{Aspectos teóricos}

El avance de la ciencia y la tecnología ha hecho que se modifiquen o actualicen los contenidos del currículo escolar tanto de la educación primaria como secundaria. No obstante, este proceso no se debería tomar a la ligera, antes de agregar un nuevo contenido al currículo escolar, se deberían analizar varios puntos importantes. Para Batanero y Godino (2001) el contenido que se va a incorporar debe ser parte de la educación general deseable para los futuros ciudadanos, ser útil para la vida posterior, ya sea para el trabajo o para el tiempo libre, ayudar al desarrollo personal y en la comprensión de los restantes temas del currículo, tanto de la educación obligatoria como superior. Para estos mismos autores estas razones son ampliamente cubiertas por las nociones estadísticas y probabilísticas, por lo que se justifica que estas temáticas sean abordadas desde la educación primaria.

Refiriéndose a estadística, Holmes (1980) apunta que debe convertirse en parte esencial de la educación general en sus primeros niveles, dado que los niños deben ser capaces de leer e interpretar tablas y gráficas que con frecuencia aparecen en los diversos medios de comunicación. Señala, además, que es útil para la vida futura porque muchas profesiones emplean al menos los conocimientos básicos de esta disciplina y por lo tanto, es 
fundamental que los estudiantes comprendan y aprecien su papel en la sociedad. Aunque ha pasado algún tiempo desde la afirmación de Holmes, el mensaje sigue vigente actualmente.

Respecto a probabilidad, según Batanero (2001), la intuición posee un papel determinante, y el diseño de actividades educativas en las que se requiera su empleo, tiene dos grandes funciones: desde edades tempranas permite que el niño comprenda el entorno por sus propios medios antes de ser capaz de entender la complejidad del modelo matemático, además, colabora con el desarrollo del conocimiento analítico que tendrá que emplear posteriormente.

Al enseñar estas disciplinas desde primaria se favorece la comprensión de otros temas del currículo y facilita la toma de decisiones. Una adecuada enseñanza de estas temáticas contribuye con la formación de personas críticas, reflexivas y analíticas.

Para conseguir que el proceso de enseñanza y aprendizaje de la probabilidad y la estadística cumplan con su labor y desarrollen individuos, que analicen condiciones y datos para tomar decisiones, se necesita que los encargados de impartir estas temáticas en las aulas estén bien preparados.

\subsection{Formación de docentes para I y II Ciclo de la Educación General Básica}

La formación recibida en la universidad es fundamental en el desempeño profesional. En el ámbito educativo, la preparación académica juega un papel fundamental en los salones de clase, pues es ahí donde los conocimientos adquiridos se ponen en práctica.

Autores como Gattuso y Panone (2002), Heaton (2002), Mendoca, Couthino y Almouloud, (2006), citados por Aparicio y Bazán (2006), mencionan que los docentes omiten impartir estos temas, y una de las razones es la falta de formación, debido en parte, a la escasa preparación con la que el profesor termina sus estudios, lo que hace que cuente con pocos recursos a la hora de dar sus clases y tienda a omitir el tema; acortarlo o, en el mejor de los casos, a presentarlo con una metodología inadecuada.

En el ámbito nacional la situación es similar, Chaves (2007) menciona que los docentes cuentan con poca preparación, no tienen formación en principios teóricos, en aplicaciones y en su enseñanza.

Este panorama, hace reflexionar acerca de la formación en estadística y probabilidad que tienen los docentes de primaria de nuestro sistema educativo. El hecho de que se omita un tema en los salones de clase, apunta a que el docente no está capacitado o no le parece un tema relevante. Alpízar et al. (2012) indican que los docentes de primaria, con los que 
trabajaron, se muestran poco satisfechos con la formación recibida en los temas tratados en este artículo; por ende su enseñanza se ve afectada por falta de dominio conceptual, ausencia de estrategias de mediación adecuadas, así como el desinterés de los docentes por enseñarlos.

Es importante señalar que en Costa Rica, las obligaciones que tiene un docente de educación primaria, exigen un sin número de actividades relacionadas con la enseñanza, que van desde dominio en conocimientos de Pedagogía, Ciencias, Matemáticas, Lengua, Historia y Geografía. Esto debido a que en ocasiones el docente debe impartir las materias de Español, Matemáticas, Ciencias y Estudios Sociales, además, de todas las labores administrativas que debe desempeñar en la institución (Alfaro, Alpízar, Morales, Ramírez y Salas, 2013).

La educación actual busca profesionales que permitan al estudiante la construcción y reconstrucción del conocimiento, que sean críticos en su labor, con una visión holística de la realidad, que sean aptos en conocimientos y comprensión de la teoría pedagógica, que posean un amplio dominio de las disciplinas que van a enseñar y que sean capaces de contextualizarlas a la realidad.

Otro punto importante es la actualización del docente en contenidos y didáctica. Los contenidos de los programas y planes de estudio de las universidades parecen no ser suficientes para que el docente desempeñe sus labores de la mejor manera, por lo que se hace necesario implementar procesos alternos de formación para los docentes en ejercicio. Al incluirse los temas de estadística y probabilidad dentro de los programas de estudio de I y II Ciclo, se vuelve una necesidad la capacitación a docentes que no recibieron formación en estos temas, con el fin de brindarles las herramientas necesarias para que el proceso de enseñanza se desarrolle apropiadamente; sin embargo esto no se ha dado, la capacitación ofrecida a sido casi nula (Alpízar et al., 2012; Barrantes et al., 2010).

El docente precisa un amplio conocimiento de técnicas o métodos para impartir los distintos tópicos. Estos métodos o técnicas deben facilitar que el estudiante visualice la amplia gama de aplicaciones que tienen las disciplinas estudiadas, con el fin de que valore el papel práctico de estas, es necesario que los ejemplos y aplicaciones que se muestran en la clase sean acordes con la realidad del estudiante.

El tener conocimiento tanto sobre el tema que se enseña cómo de la metodología apropiada influye en la actitud del docente y por ende en la forma de abordar los temas en el aula. En los resultados de Aparicio y Bazán (2006) se menciona que cuando existe mayor 
conocimiento hay un cambio positivo en las actitudes. Ya que cuando esto ocurre se desarrolla un sentir, creer o pensar con sentimientos afectivos respecto a un objeto o situación. Estos autores dejar ver la importancia de los aspectos afectivos desde la perspectiva del profesor que puede tener impacto en el aprendizaje de sus estudiantes.

Conocer las actitudes de los docentes es de gran relevancia, ya que están íntimamente relacionadas con su rol como educador. Puesto que, en muchas ocasiones una actitud negativa hacia cierto tema es el resultado de una serie de factores, como lo son la poca comprensión o desconocimiento de contenidos, ignorancia de la importancia y aplicación del mismo, entre otras; con el agravante que puede trasmitir a sus estudiantes las actitudes negativas que posea. Por otra parte, una actitud positiva hacia la materia beneficia el proceso de enseñanza.

En resumen, existen diferentes factores que pueden afectar de manera positiva o negativa la enseñanza de la estadística y la probabilidad, tales como: poca formación, actitud de los docentes, conocimiento de estrategias metodológicas, entre otras, son algunos de estos factores.

El papel que tiene el docente es sumamente importante en el proceso de enseñanza y aprendizaje, razón por la cual el conocer sus actitudes hacia los temas en estudio es fundamental, ya que la percepción que tenga está relacionada con la compresión de los contenidos y el uso de una metodología adecuada en el aula.

\section{Marco metodológico}

En este documento se describe la actitud de un grupo de docentes de primera enseñanza hacia la estadística y probabilidad.

La investigación realizada para generar el presente documento, se puede catalogar como no experimental descriptiva, esto pues de acuerdo con Hernández, Fernández y Baptista (2006) se describen hechos que sucedieron y el estudio busca generar las interpretaciones correspondientes a esos hechos. Los investigadores se mantuvieron al margen de los sucesos para determinar y describir actitudes del grupo de sujetos en estudio.

\subsection{Sujetos: población y muestra}

La población estuvo constituida por docentes de I y II Ciclo de la Educación General Básica, de escuelas públicas de la Dirección Regional Educativa de Heredia. Esta dirección está compuesta por cinco circuitos, de la cual se tomó una muestra de 20 docentes, donde 
hay representación de los cinco circuitos. La muestra se seleccionó por conveniencia, los maestros fueron elegidos por los asesores pedagógicos de Matemática de Heredia, para asistir a un curso de capacitación relacionada con didáctica de la probabilidad y la estadística para primaria.

Los criterios de selección que utilizaron los asesores para elegir a los docentes participantes fue el interés de estos por capacitarse y el compromiso que habían mostrado con la asesoría regional al convocarlo a actividades anteriores, no se había realizado con ellos ninguna actividad relacionadas con los temas tratados en este artículo. Es importante aclarar que al ser una muestra tomada por conveniencia (no aleatoria) los resultados obtenidos reflejan la realidad del grupo estudiado, no se pueden inferir resultados a la población.

Los participantes asistieron a un curso de capacitación brindado por la Escuela de Matemática de la Universidad Nacional en conjunto con la Dirección Regional de Heredia, dicho curso estuvo orientado a la didáctica de la probabilidad y la estadística en educación primaria y tuvo una duración de 60 horas.

Se abordaron temas como: historia e importancia de la probabilidad y la estadística, tipos de datos y tipos de variables, recolección de datos, representación de datos (tabular y gráfica), medidas estadísticas, conceptos básicos de probabilidad, además de didácticas especificas para abordar estos temas en el aula.

Es importante aclarar que aunque no se pueden generalizar los resultados que se obtuvieron, al usar una muestra no probabilística, la descripción de ellos podría ser un insumo valioso para conocer el estado de la enseñanza de la probabilidad y la estadística a nivel de primaria.

\subsection{Técnicas de recolección de información}

Para la recolección de la información se hizo uso del cuestionario autoadministrado. El objetivo del cuestionario fue recolectar las actitudes de los docentes hacia la estadística y la probabilidad como áreas del saber y también en su proceso de enseñanza y aprendizaje. El cuestionario estuvo conformado por ítems relacionados con la importancia, utilidad y dificultad de las temáticas en cuestión.

El instrumento estuvo basado en el cuestionario utilizado por Estrada (2002) sobre las actitudes del profesorado hacia estadística y probabilidad; se contextualizaron las preguntas al medio donde se iba a aplicar y se agregaron algunas construidas por estadísticos de la 
Universidad Nacional. La validación se realizó con la colaboración de un grupo de diez maestras, las cuales no pertenecen a la muestra en estudio, a las cuales se les aplicó el cuestionario; además el coeficiente de alfa de Cronbach, sobre los casos válidos, sobrepasa 0,9 lo que indica una consistencia interna de los ítems y la fiabilidad del instrumento.

El cuestionario estuvo dividido en dos apartados. El primero abarcó aspectos personales como: sexo, nombre del centro o los centros educativos donde labora, categoría profesional, grado o los grados académicos alcanzados, año en que lo obtuvo, institución donde estudió y nombre del título obtenido, años de experiencia, los niveles o grados que tenían a cargo. El segundo apartado contiene 32 proposiciones que pretenden conocer la actitud de los docentes hacia las disciplinas de interés, se utilizó la escala tipo Likert donde 1 significaba estar muy en desacuerdo con la proposición dada, 2 en desacuerdo, 3 indiferente, 4 de acuerdo y 5 muy de acuerdo.

Luego de la recolección de datos, estos se organizaron, tabularon y analizaron, por medio de técnicas de estadística descriptiva, lo que permitió generar conclusiones y recomendaciones.

El cuestionario se aplicó al inicio y al final de la capacitación con el fin de determinar si existían diferencias en la forma de pensar de los docentes después de un proceso de socialización de los temas estudiados.

\section{Análisis de datos}

\subsection{Descripción de los participantes}

Los docentes que participaron en el estudio pertenecían a la Dirección Regional Educativa de Heredia, 18 laboraban en escuelas diurnas públicas, impartían lecciones de I y II Ciclo de la Educación General Básica y particularmente Matemáticas; y dos asesores pedagógicos de Matemática del MEP.

De los 20 participantes, solamente habían dos varones. Cinco docentes poseen grado académico de maestría obtenido en universidades privadas y el resto con licenciatura (algunas estatales y otras privadas), 11 afirman haber recibido en su formación profesional cursos donde se impartieron tópicos de probabilidad y estadística. 76,6\% de los encuestados tienen seis o más años de experiencia. Además, 15 poseen la categoría profesional mayor para su gremio PT6. Lo anterior permite suponer que estos docentes poseen la preparación adecuada para desempeñar su labor, ya que casi todos cuentan con la máxima categoría profesional y tienen experiencia laboral. 


\subsection{Actitudes iniciales}

Estas creencias o actitudes fueron medidas por medio de un cuestionario aplicado durante la primera sesión de una capacitación de cinco. Fue lo primero que se realizó en dicho evento por lo que los participantes no estaban influenciados con las temáticas a tratar.

El cuestionario tenía 32 proposiciones, algunas preguntas positivas y otras negativas, para el análisis respectivo se separaron en esas dos categorías.

En cada uno de los cuadros donde se presentan las proposiciones se utilizó simbología que se define a continuación: para la moda Mo, para la media $\bar{x}$, para la desviación estándar $s, M e$ para la mediana y para el total de docentes $n$. Se establecieron 3 categorías con la escala del cuestionario; a saber: MD son los docentes que contestaron estar muy en desacuerdo o en desacuerdo, I son los que se mostraron indiferente ante la proposición y MDA los que expresaron estar muy de acuerdo o de acuerdo con lo planteado, estas categorías se incluyen en los cuadros estadísticos respectivos. Los porcentajes están basados en la totalidad de personas que contestaron cada ítem, el cual se muestra en la última columna de cada cuadro.

\subsubsection{Análisis de proposiciones negativas}

Cabe acotar aquí que según la escala del cuestionario, la puntuación máxima (5) significaba estar muy de acuerdo con la proposición enunciada y la puntuación mínima (1) estar en total desacuerdo. 


\section{Cuadro 1 \\ Percepciones iniciales hacia la estadística y probabilidad de los docentes encuestados, cuando las proposiciones estuvieron redactadas en forma negativa. Dirección Regional de Heredia,} 2011

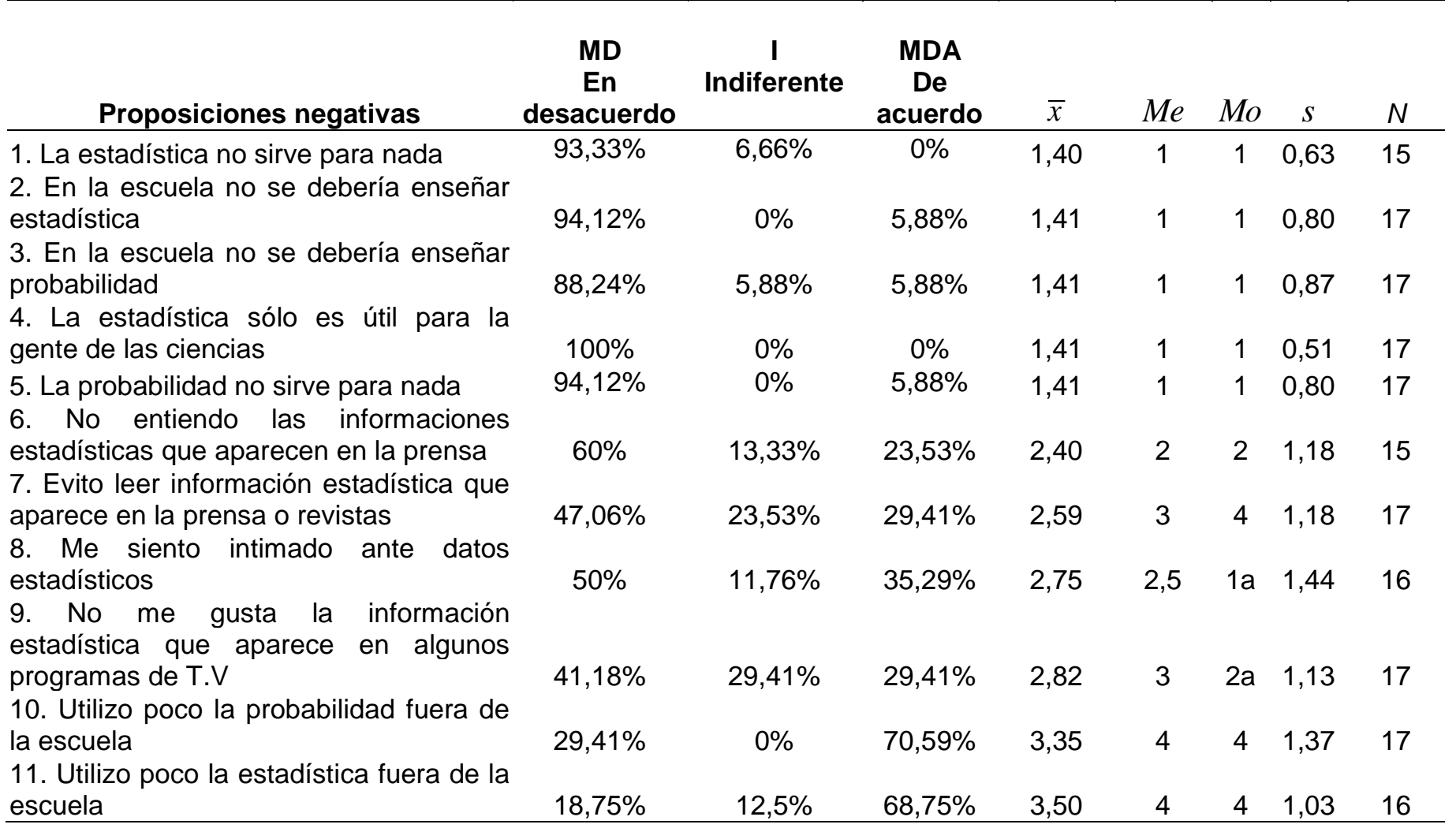

a: Hay varias modas pero se muestra la menor de ellas.

Fuente: Elaboración propia (2011).

Del cuadro 1 se puede determinar que de los participantes 93,33\% muestran su total desacuerdo ante la proposición la Estadística no sirve para nada, de manera similar que en el ítem la Probabilidad no sirve para nada, con 94,12\% en desacuerdo. Además muchos de ellos consideran que estas áreas del saber son útiles para la vida y no solo para las personas relacionadas con las ciencias, esto se deduce al tener media de aproximadamente 1 y una desviación estándar baja en el ítem la Estadística sólo es útil para la gente de ciencias. Ante la proposición no se debería enseñar Estadística en la escuela, gran parte de los docentes muestran su desacuerdo. En términos generales los participantes de este estudio coincidieron que la probabilidad y la estadística son útiles y deben enseñarse en primaria.

Para el ítem utilizo poco la probabilidad fuera de la escuela, se obtuvo una media de 3,35 y por tanto los datos revelan que los docentes se muestran indiferentes o de acuerdo 
respecto al poco uso que se le da. Situación similar ocurre ante la afirmación utilizo poco la estadística fuera de la escuela, donde $68,75 \%$ de los participantes están de acuerdo con esto.

En los ítems, No me gusta la información estadística que aparece en algunos programas de T.V y Evito leer información estadística que aparece en la prensa o revistas, la mediana es 3 y el promedio se acerca a ese valor, por lo que se puede notar cierta indiferencia por parte de los participantes, sin embargo la desviación estándar, para ambos ítems es alta (mayor a uno) lo que muestra que hay variabilidad en las respuestas y por tanto las opiniones de a favor o en contra están divididas. Cabe destacar que la moda en el ítem Evito leer información estadística que aparece en la prensa o revistas es 4, la respuesta con mayor frecuencia.

La información recolectada muestra que, en general, los docentes no se sienten intimidados por los datos estadísticos, ya que ante la frase me siento intimidado ante datos estadísticos la media es 2,75 y la mediana 2,5.

En general, los participantes consideran que la estadística y la probabilidad son útiles para la vida y que se deben enseñar desde primaria a los estudiantes, aunque admiten que las utilizan poco fuera de la escuela e inclusive evitan la información que se relacione con ellas fuera del salón de clase. Esto indica que aunque consideran que son disciplinas de importancia no logran utilizarlas en situaciones cotidianas, lo cual puede influir en su apatía de leer información estadística que presentan diferentes medios de comunicación.

A continuación se presenta el análisis de los ítems redactados en forma positiva.

\subsubsection{Análisis de preguntas positivas}

En el cuadro 2 se encentran las estadísticas relacionadas con los ítems positivos. 
Cuadro 2

Percepciones iniciales hacia la estadística y probabilidad de los docentes encuestados, cuando las proposiciones estuvieron redactadas en forma positiva. Dirección Regional de Heredia, 2011

\begin{tabular}{|c|c|c|c|c|c|c|c|c|}
\hline Proposiciones positivas & $\begin{array}{c}\text { MD } \\
\text { En } \\
\text { desacuer } \\
\text { do }\end{array}$ & $\begin{array}{c}\text { I } \\
\text { Indiferent } \\
\mathrm{e}\end{array}$ & $\begin{array}{l}\text { MDA } \\
\text { De } \\
\text { acuerdo }\end{array}$ & $\bar{x}$ & $M e$ & $M o$ & $S$ & $N$ \\
\hline $\begin{array}{l}\text { 1. Se debería eliminar la Probabilidad del } \\
\text { currículo de primaria }\end{array}$ & $94 \%$ & $6 \%$ & $0 \%$ & 1,4 & 1 & 1 & 0,61 & 17 \\
\hline $\begin{array}{l}\text { 2. Se debería eliminar la Estadística del } \\
\text { currículo de primaria }\end{array}$ & $94 \%$ & $0 \%$ & $6 \%$ & 1,4 & 1 & 1 & 0,80 & 17 \\
\hline $\begin{array}{l}\text { 3. La Probabilidad es fácil } \\
\text { 4.Me gusta resolver problemas cuando }\end{array}$ & $25 \%$ & $25 \%$ & $50 \%$ & 3,3 & 3,5 & 4 & 1,06 & 16 \\
\hline $\begin{array}{l}\text { uso la Estadística } \\
5 \text {. Los problemas de Probabilidad me }\end{array}$ & $18 \%$ & $18 \%$ & $65 \%$ & 3,5 & 4 & 4 & 1,01 & 17 \\
\hline resultan fáciles & $35 \%$ & $0 \%$ & $65 \%$ & 3,5 & 4 & 4 & 1,18 & 17 \\
\hline $\begin{array}{l}\text { 6. La Estadística es fácil } \\
\text { 7. Disfruto resolver problemas donde } \\
\text { están involucrados conceptos de }\end{array}$ & $18 \%$ & $18 \%$ & $65 \%$ & 3,7 & 4 & 4 & 1,05 & 17 \\
\hline Probabilidad & $12 \%$ & $18 \%$ & $71 \%$ & 3,7 & 4 & 4 & 0,85 & 17 \\
\hline $\begin{array}{l}\text { 8. Los problemas de Estadística me } \\
\text { resultan fáciles }\end{array}$ & $29 \%$ & $6 \%$ & $65 \%$ & 3,7 & 4 & 4 & 1,22 & 17 \\
\hline $\begin{array}{l}\text { 9. Me agrada la Probabilidad porque } \\
\text { ayuda a ver los problemas objetivamente }\end{array}$ & $12 \%$ & $6 \%$ & $82 \%$ & 3,9 & 4 & 4 & 0,86 & 17 \\
\hline decisiones más documentadas & $13 \%$ & $0 \%$ & $88 \%$ & 4,0 & 4 & 4 & 0,89 & 16 \\
\hline 11. Disfruto enseñar Estadística & $6 \%$ & $12 \%$ & $82 \%$ & 4,1 & 4 & 4 & 0,86 & 17 \\
\hline $\begin{array}{l}\text { 12. Disfruto enseñar Probabilidad } \\
\text { 13. Uso la Estadística para resolver }\end{array}$ & $12 \%$ & $6 \%$ & $82 \%$ & 4,1 & 4 & 4 & 0,97 & 17 \\
\hline $\begin{array}{l}\text { problemas de la vida cotidiana } \\
\text { 14. La Probabilidad es fundamental en la }\end{array}$ & $6 \%$ & $6 \%$ & $88 \%$ & 4,1 & 4 & 4 & 0,78 & 17 \\
\hline $\begin{array}{l}\text { formación básica del ciudadano } \\
\text { 15. Me agrada la Estadística porque }\end{array}$ & $0 \%$ & $6 \%$ & $94 \%$ & 4,3 & 4 & 4 & 0,61 & 17 \\
\hline $\begin{array}{l}\text { ayuda a ver los problemas objetivamente } \\
\text { 16. Encuentro interesante el mundo de la }\end{array}$ & $0 \%$ & $0 \%$ & $100 \%$ & 4,3 & 4 & 4 & 0,45 & 16 \\
\hline Estadística & $0 \%$ & $6 \%$ & $94 \%$ & 4,41 & 4 & 4 & 0,61 & 17 \\
\hline $\begin{array}{l}\text { 17. Me gustan los trabajos donde } \\
\text { aparecen estudios estadísticos }\end{array}$ & $0 \%$ & $6 \%$ & $94 \%$ & 4,41 & 4 & $4 a$ & 0,62 & 17 \\
\hline $\begin{array}{l}\text { 18. La Estadística ayuda a tomar } \\
\text { decisiones más documentadas } \\
\text { 19. Por medio de la Estadística se puede }\end{array}$ & $0 \%$ & $6 \%$ & $94 \%$ & 4,41 & 4 & $4 a$ & 0,62 & 17 \\
\hline $\begin{array}{l}\text { manipular la realidad } \\
\text { 20. La Estadística es fundamental en la }\end{array}$ & $0 \%$ & $12 \%$ & $88 \%$ & 4,5 & 5 & 5 & 0,72 & 17 \\
\hline $\begin{array}{l}\text { información básica del ciudadano } \\
\text { 21. La Estadística ayuda a entender el }\end{array}$ & $0 \%$ & $0 \%$ & $100 \%$ & 4,53 & 5 & 5 & 0,51 & 17 \\
\hline mundo & $0 \%$ & $0 \%$ & $100 \%$ & 4,65 & 5 & 5 & 0,49 & 17 \\
\hline
\end{tabular}

a: Hay varias modas pero se muestra la menor de ellas.

Fuente: Elaboración propia (2011). 
Casi la totalidad de los docentes muestran su desacuerdo en que se elimine la probabilidad y la estadística del programa de estudios de primaria, esto se refleja con las medidas estadísticas obtenidas en los enunciados correspondientes a se debería eliminar la Probabilidad del currículo de primaria y se debería eliminar la Estadística del currículo de primaria con valores de media con 1,4; la mediana con 1 y la moda un 1 en ambos ítems. El 94\% está de acuerdo con la afirmación que dice La probabilidad es fundamental en la formación básica del ciudadano, su desviación estándar es una de las más bajas $(0,61)$, esto indica que la posición de los participantes es concordante entre ellos. Sin embargo, un participante se mostró de acuerdo con la eliminación de estas áreas del currículo lo cual es preocupante porque son fundamentales para el desarrollo de un ciudadano responsable de sus decisiones.

En cuanto al enunciado La probabilidad es fácil, cuatro de los participantes están en desacuerdo, asimismo en el ítem La estadística es fácil tres opinaron estar en desacuerdo con la proposición. Esto evidencia que a cierto número de docentes les resulta difícil algunos de los tema tratados, por lo que se debe presentar atención pues una actitud negativa por parte del educador puede incidir en la actitud de los educandos; además, si tienen dificultades podrían presentar limitaciones en el desarrollo de las lecciones referidas a estos temas.

En cuanto a la facilidad con la que perciben los conceptos sus opiniones son similares para ambos temas, se encuentran en el nivel intermedio; pero, ambas desviaciones estándar son altas (1,05 y 1,06 respectivamente) lo que indica que las opiniones en estos aspectos estuvieron divididas, la estadística es considerada fácil por más docentes (65\%) en relación con la probabilidad (50\%), esto puede deberse a la falta de formación profesional y a la ausencia de capacitación, lo cual los hace sentirse temerosos hacia los conceptos teóricos de las mismas.

En el ítem Uso la estadística para resolver problemas de la vida cotidiana, 88\% está de acuerdo con la proposición y en Me gusta resolver problemas cuando uso la estadística 65\% lo están. Los participantes manifiestan que si se les presenta algún problema en la vida cotidiana usan la estadística como herramienta para resolverlo, por otra parte; admiten que la utilizan poco fuera de la escuela e inclusive evitan la información que se relacione con estas disciplinas, por lo que se encuentra aquí una contradicción en sus respuestas.

A pesar que $25 \%$ de los participantes no les resulta fácil la probabilidad, a la mayoría les agrada y disfrutan resolver problemas que involucran estos temas y que les ayudan, para 
resolverlos objetivamente; esto se refleja con las medidas estadísticas obtenidas en el enunciado Me agrada la probabilidad porque ayuda a ver los problemas objetivamente con una media de 3,9, una moda y mediana de 4 y una desviación estándar de 0,86.

Los participantes consideran que la estadística ayuda a tomar decisiones más documentadas, su media fue de 4,41 y su desviación estándar una de las más bajas $(0,62)$. En cuanto a probabilidad $13 \%$ considera que ayuda a tomar decisiones más documentadas aunque su media fue menor que el ítem relacionado con estadística con un 4,0. 82\% dicen disfrutar enseñar los temas en estudio; además, 100\% de los encuestados se muestran entre de acuerdo y muy de acuerdo ante la afirmación me agrada la Estadística porque ayuda a ver los problemas objetivamente, siendo la moda cuatro; mostrando con estos datos una actitud positiva hacia estas disciplinas.

Por otra parte, los docentes muchas veces consideran que existen temas dentro del currículo que no son tan relevantes, esta actitud hacia un tema particular es trasmitida a los estudiantes, si son de primaria la actitud de su docente los marcará por el resto de su vida académica, por lo que fue muy importante conocer si los participantes les gusta impartir las temáticas tratadas, la media obtenida en las proposiciones Disfruto enseñar estadística y Disfruto enseñar probabilidad fue mayor a cuatro, en ambas proposiciones, lo que indica que a los docentes les gusta impartir lecciones de estos temas, solo tres de ellos no disfrutan o se muestran indiferentes, aun así se debe prestar atención a esta situación pues el docente podría estar influenciando al educando de manera negativa tanto en su componente afectivo (el sentir interés hacia la misma) como en el componente cognitivo (pensar que tiene suficientes conocimientos y habilidades) y de esta manera considerar la materia como fácil o difícil.

En cuanto a si les gustan los trabajos en donde aparecen estudios estadísticos y si encuentran interesante el mundo de la estadística; la moda y mediana coinciden dando una puntuación muy positiva (4), las desviaciones estándar en estos ítems son bajas lo que demuestra que la variabilidad de las respuesta no fue mucha.

Un ítem con una media alta fue Por medio de la estadística se puede manipular la realidad, además su mediana y moda de cinco indican que un alto número de encuestados está a favor, la variabilidad en este caso es bastante baja. Esto puede deberse a diferentes situaciones vividas, como ejemplo las experiencias que se han tenido en Costa Rica y otros países acerca de las encuestas políticas donde se han dado resultados en las elecciones 
presidenciales muy distintos a los pronosticados por encuestas realizadas días antes de la elección.

Otro ítem que posee una de las medias más altas fue La estadística ayuda a entender el mundo con una media de 4,65, tanto la moda como la mediana fue de cinco y una desviación estándar de 0,49; esto indica que los participantes sienten que la estadística les ayuda a entender el mundo que los rodea, además de ser una herramienta que les sirve para poder interpretar algunos fenómenos que se presentan en la vida cotidiana; sin embargo algunos participantes admiten que la utilizan poco fuera de la escuela.

Se debe rescatar lo que surge en estos ítems ya que se contradicen al estar de acuerdo con los dos anteriores, pues están a favor que con la estadística se puede manipular la realidad y a la vez manifestar que les ayuda a entender el mundo que los rodea. Por último, para el enunciado La estadística es fundamental en la formación básica del ciudadano, se obtuvo una media de 4,53, lo que establece que los participantes son conscientes que está temática debe formar parte del currículo escolar.

En conclusión, se evidencia una actitud positiva hacia estadística y probabilidad. En estos ítems al igual que en los negativos la mayoría de participantes reconocen la importancia de impartirlos desde primaria, ya que son fundamentales en la formación básica de una persona, aunque una parte de ellos no las consideran fáciles, razón que puede influir en no impartir el tema o brindarle menos tiempo e importancia.

Respecto al uso que se les da en la vida, encontramos una contradicción entre los ítems negativos y positivos, pues en los negativos admiten que no utilizan las disciplinas fuera de la escuela y en los positivos, como se mencionó, 88\% dicen utilizar la Estadística en su vida cotidiana. Por otra parte, debemos dejar en claro que puede ser que los docentes no sean conscientes que utilizan la estadística en muchas situaciones y por tanto el ítem no fue claro para los participantes.

Aunque en general se evidencia una actitud positiva, algunos participantes consideran que los temas son difíciles de entender e inclusive prefieren ignorar la información estadística y probabilística que se presenta en los diferentes medios de comunicación, admitiendo que no la utilizan en la vida cotidiana, sino, únicamente, en problemas realizados dentro de las aulas, situación preocupante, ya que ellos son los encargados de enseñar estos temas; además de crear y fomentar un ambiente agradable para el aprendizaje de estos inculcándoles que son una herramienta que les permitirá resolver problemas en la vida cotidiana. 


\subsection{Percepciones finales}

Después del proceso de capacitación se aplicó el mismo cuestionario, con el fin de comparar las actitudes que tuvieron al inicio y al final de dicho proceso.

Al igual que el análisis del primer cuestionario se separaron los ítems positivos de los negativos y se presenta la información de la misma manera.

\subsubsection{Análisis de ítems negativos}

Cabe recordar aquí que la puntuación máxima en la escala (5) significa estar de acuerdo con la proposición enunciada, las cuales están redactadas en sentido negativo. En el cuadro 3 adjunto se presentan algunas medidas estadísticas obtenidas de las respuestas dadas por los docentes.

\section{Cuadro 3}

Percepciones finales hacia la estadística y probabilidad de los docentes encuestados, cuando las proposiciones estuvieron redactadas en forma negativa. Dirección Regional de Heredia, 2011

\begin{tabular}{|c|c|c|c|c|c|c|c|c|}
\hline Proposiciones negativas & $\begin{array}{c}\text { MD } \\
\text { En } \\
\text { desacuerd } \\
0\end{array}$ & $\begin{array}{l}\text { I } \\
\text { Indiferente }\end{array}$ & $\begin{array}{l}\text { MDA } \\
\text { De } \\
\text { acuerdo }\end{array}$ & $\bar{x}$ & $M e$ & Mo & $S$ & $N$ \\
\hline 1. La estadística no sirve para nada & $100 \%$ & $0 \%$ & $0 \%$ & 1,0 & 1 & 1 & 0,00 & 17 \\
\hline $\begin{array}{l}\text { 2. En la escuela no se debería enseñar } \\
\text { estadística } \\
\text { 3. En la escuela no se debería enseñar }\end{array}$ & $100 \%$ & $0 \%$ & $0 \%$ & 1,0 & 1 & 1 & 0,00 & 16 \\
\hline probabilidad & $100 \%$ & $0 \%$ & $0 \%$ & 1,1 & 1 & 1 & 0,25 & 16 \\
\hline $\begin{array}{l}\text { 4. La probabilidad no sirve para nada } \\
\text { 5. La estadística sólo es útil para la }\end{array}$ & $82 \%$ & $0 \%$ & $18 \%$ & 1,7 & 1 & 1 & 1,46 & 17 \\
\hline $\begin{array}{l}\text { gente de las ciencias } \\
6 \text {. Evito leer información estadística que }\end{array}$ & $94 \%$ & $0 \%$ & $6 \%$ & 1,3 & 1 & 1 & 1,00 & 17 \\
\hline $\begin{array}{l}\text { aparece en la prensa o revistas } \\
\text { 7. No entiendo las informaciones }\end{array}$ & $65 \%$ & $29 \%$ & $6 \%$ & 2,1 & 2 & 1 & 1,14 & 17 \\
\hline estadísticas que aparecen en la prensa & $70 \%$ & $18 \%$ & $12 \%$ & 2,2 & 2 & 2 & 0,90 & 17 \\
\hline $\begin{array}{l}\text { 8. No me gusta la información } \\
\text { estadística que aparece en algunos } \\
\text { programas de T.V }\end{array}$ & $6 \%$ & $31 \%$ & $63 \%$ & 3,5 & 4 & 4 & 0,82 & 16 \\
\hline $\begin{array}{l}\text { 9. Utilizo poco la estadística fuera de la } \\
\text { escuela } \\
\text { 10. Utilizo poco la probabilidad fuera de }\end{array}$ & $18 \%$ & $53 \%$ & $29 \%$ & 3,1 & 3 & 3 & 0,93 & 17 \\
\hline la escuela & $23 \%$ & $53 \%$ & $24 \%$ & 3,0 & 3 & 3 & 0,94 & 17 \\
\hline estadísticos & $65 \%$ & $23 \%$ & $12 \%$ & 2,3 & 2 & 2 & 1,11 & 17 \\
\hline
\end{tabular}

a: Hay varias modas pero se muestra la menor de ellas.

Fuente: Elaboración propia (2011). 
Según el cuadro 3 la totalidad de los participantes está en desacuerdo con las afirmaciones de que la Estadística no sirve para nada y que en la escuela no se debería enseñar dicha temática, lo cual es muy positivo. La mayoría está en desacuerdo con que la estadística sólo es útil para la gente de ciencias lo que demuestra que comprenden la utilidad de esta disciplina en diferentes campos.

En cuanto a probabilidad también casi todos los docentes consideran que es importante, ayuda en la cotidianidad y se debe enseñar en la escuela. Esto se ve reflejado en el ítem en la escuela no se debería enseñar probabilidad, ya que $100 \%$ de los participantes se muestran en desacuerdo o muy en desacuerdo ante esta afirmación, y ante la probabilidad no sirve para nada, solo $18 \%$ está de acuerdo, aunque este último porcentaje sea bajo, es preocupante pensar que existen docentes que consideran que uno de los temas que enseñan a sus estudiantes, no sirve para nada.

El promedio de las opiniones relacionadas con proposiciones como evitar leer información estadística y la incomprensión de los datos que se presentan en algunos medios de comunicación es bastante bajo, alrededor de dos, lo que indica que los docentes no están de acuerdo con dichos enunciados, presentando una leve mejoría en cuanto al cuestionario inicial; sin embargo, en cuanto al gusto por la información estadística de los programas de televisión más de la mitad de los participantes expresaron disgustarle, lo cual presenta una contradicción entre sus percepciones o creencias.

En cuanto al uso que le dan a las áreas fuera del salón de clase, se nota una mejoría en el cuestionario final; sin embargo siguen estando los promedios, medianas y modas alrededor de tres mostrando cierta indiferencia por parte de los participantes.

Por tanto, en los ítems redactados en forma negativa se evidencia que, la mayoría de, los docentes consideran que los conocimientos en estadística y probabilidad son importantes en la formación de un individuo y que se deben enseñar desde primaria, aún así, a algunos les sigue disgustando la información estadística presentada en diferentes medios de comunicación y continúan manifestando indiferencia en el uso que se les da a estas temáticas fuera de la escuela.

\subsubsection{Análisis de ítems positivos}

A continuación se presenta el análisis de los ítems redactados en forma positiva. 


\section{Cuadro 4 \\ Percepciones finales hacia la estadística y probabilidad de los docentes encuestados, cuando las proposiciones estuvieron redactadas en forma positiva. Dirección Regional de Heredia, 2011}

\begin{tabular}{|c|c|c|c|c|c|c|c|c|}
\hline Proposiciones positivas & $\begin{array}{l}\text { MD } \\
\text { En } \\
\text { desacuerdo }\end{array}$ & $\stackrel{\text { I }}{\text { Indiferente }}$ & $\begin{array}{l}\text { MDA } \\
\text { De } \\
\text { acuerdo }\end{array}$ & $\bar{x}$ & $M e$ & Mo & $s$ & $N$ \\
\hline $\begin{array}{l}\text { 1. Se debería eliminar la Probabilidad del } \\
\text { currículo de primaria. }\end{array}$ & $100 \%$ & $0 \%$ & $0 \%$ & 1,1 & 1 & 1 & 0,24 & 17 \\
\hline currículo de primaria. & $100 \%$ & $0 \%$ & $0 \%$ & 1,1 & 1 & 1 & 0,00 & 16 \\
\hline $\begin{array}{l}\text { 3. La Probabilidad es fácil. } \\
\text { 4. Los problemas de Probabilidad me }\end{array}$ & $29 \%$ & $29 \%$ & $41 \%$ & 3,2 & 3 & 4 & 0,95 & 17 \\
\hline resultan fáciles. & $12 \%$ & $41 \%$ & $47 \%$ & 3,4 & 3 & $3 a$ & 0,93 & 17 \\
\hline $\begin{array}{l}\text { 5. La Estadística es fácil. } \\
\text { 6. Los problemas de Estadística me }\end{array}$ & $24 \%$ & $24 \%$ & $53 \%$ & 3,5 & 4 & 4 & 1,10 & 17 \\
\hline resultan fáciles. & $13 \%$ & $31 \%$ & $57 \%$ & 3,6 & 4 & 4 & 0,96 & 16 \\
\hline $\begin{array}{l}\text { 7. Disfruto enseñar Probabilidad } \\
\text { 8.Me gusta resolver problemas cuando }\end{array}$ & $6 \%$ & $24 \%$ & $70 \%$ & 3,9 & 4 & 4 & 0,90 & 17 \\
\hline uso la Estadística & $0 \%$ & $12 \%$ & $88 \%$ & 3,9 & 4 & 4 & 0,92 & 15 \\
\hline $\begin{array}{l}\text { 9. Uso la Estadística para resolver } \\
\text { problemas de la vida cotidiana }\end{array}$ & $6 \%$ & $18 \%$ & $77 \%$ & 3,9 & 4 & 4 & 1,00 & 17 \\
\hline $\begin{array}{l}\text { 10. La Probabilidad ayuda a tomar } \\
\text { decisiones más documentadas. } \\
\text { 11. Disfruto resolver problemas donde } \\
\text { están involucrados conceptos de }\end{array}$ & $12 \%$ & $18 \%$ & $70 \%$ & 4,0 & 4 & 5 & 1,10 & 17 \\
\hline Probabilidad & $0 \%$ & $18 \%$ & $82 \%$ & 4,1 & 4 & 4 & 0,70 & 17 \\
\hline 12. Disfruto enseñar Estadística & $6 \%$ & $18 \%$ & $76 \%$ & 4,1 & 4 & 5 & 0,93 & 17 \\
\hline $\begin{array}{l}\text { 13. La Estadística ayuda a tomar } \\
\text { decisiones más documentadas } \\
\text { 14. La Probabilidad es fundamental en la }\end{array}$ & $0 \%$ & $19 \%$ & $82 \%$ & 4,2 & 4 & 4 & 0,75 & 16 \\
\hline $\begin{array}{l}\text { formación básica del ciudadano } \\
\text { 15. Por medio de la Estadística se puede }\end{array}$ & $0 \%$ & $18 \%$ & $82 \%$ & 4,2 & 4 & $4^{\mathrm{a}}$ & 0,75 & 17 \\
\hline $\begin{array}{l}\text { manipular la realidad } \\
\text { 16. Me agrada la Probabilidad porque }\end{array}$ & $6 \%$ & $12 \%$ & $82 \%$ & 4,2 & 4 & 5 & 0,90 & 17 \\
\hline $\begin{array}{l}\text { ayuda a ver los problemas objetivamente } \\
\text { 17. Me gustan los trabajos donde }\end{array}$ & $0 \%$ & $19 \%$ & $82 \%$ & 4,3 & 4 & 5 & 0,78 & 16 \\
\hline $\begin{array}{l}\text { aparecen estudios estadísticos. } \\
\text { 18. La Estadística es fundamental en la }\end{array}$ & $0 \%$ & $12 \%$ & $88 \%$ & 4,4 & 5 & 5 & 0,71 & 17 \\
\hline $\begin{array}{l}\text { formación básica del ciudadano } \\
\text { 19. Me agrada la Estadística porque }\end{array}$ & $6 \%$ & $0 \%$ & $94 \%$ & 4,4 & 5 & 5 & 1,00 & 17 \\
\hline $\begin{array}{l}\text { ayuda a ver los problemas objetivamente } \\
20 \text {. Encuentro interesante el mundo de la }\end{array}$ & $0 \%$ & $13 \%$ & $87 \%$ & 4,5 & 5 & 5 & 0,72 & 17 \\
\hline Estadística & $0 \%$ & $6 \%$ & $94 \%$ & 4,6 & 5 & 5 & 0,62 & 16 \\
\hline 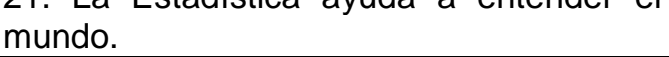 & $0 \%$ & $0 \%$ & $100 \%$ & 4,8 & 5 & 5 & 0,44 & 17 \\
\hline
\end{tabular}

a: Hay varias modas pero se muestra la menor de ellas.

Fuente: Elaboración propia (2011). 
De acuerdo con los datos del cuadro 4 la totalidad de los docentes no están de acuerdo con eliminar la probabilidad y la estadística del currículo de primaria.

En cuanto a la facilidad con la que perciben las disciplinas, las opiniones siguen estando divididas; pero, puede notarse una pequeña mejoría en cuanto a la percepción de la facilidad con la que ven la estadística y problemas relacionados con esta. El gusto por enseñar es un poco mayor en estadística que en probabilidad, su promedio superó los cuatro puntos, la moda fue cinco y la desviación estándar es baja. Este es un aspecto que debe trabajarse aún más en procesos de formación y capacitación ya que si un docente no se siente cómodo al impartir sus lecciones puede trasmitirle actitudes negativas a sus estudiantes.

En las afirmaciones Encuentro interesante el mundo de la estadística y la estadística ayuda a entender el mundo la mayoría de los participantes se muestran de acuerdo, siendo estos los ítems con mayor promedio; además la moda fue cinco y las desviaciones estándar son bajas, lo que muestra que no hubo mucha variabilidad en las respuestas.

Existe un mayor porcentaje (94\%) de participantes que opinan que La Estadística es fundamental en la formación básica del ciudadano, por otra parte ante la afirmación La Probabilidad es fundamental en la formación básica del ciudadano (82\%) aunque en ambos ítems los promedios son mayores a cuatro, la mediana y moda respecto a estadística es mayor; esto puede deberse a que se está más acostumbrado a un acercamiento con conceptos estadísticos como la representación de datos (cuadros y gráficas), mientras que los probabilísticos son más abstractos como la toma de decisiones en ambientes de incertidumbre o el azar. Sumado a esto la mayoría de los docentes consideran que las áreas en estudio ayudan a ver los problemas cotidianos de manera más objetiva.

Siguen considerando que por medio de la estadística se puede manipular la realidad, aunque la media es menor a la del primer cuestionario, como se citó anteriormente esto puede deberse a la influencia que tenemos de las encuestas políticas o por medio de los trucos visuales que se les pueden hacer a las representaciones gráficas para adulterar la información que se desea proyectar; sin embargo la mayoría considera que gracias a los métodos estadísticos y probabilísticos se pueden tomar decisiones más documentadas.

La utilidad e importancia que tienen estas disciplinas es reconocida por los docentes pues un porcentaje alto opinó que estos son temas fundamentales en la formación básica del ciudadano, aunque concuerdan en que no siempre es sencillo entender los procedimientos y la información que aparece en los diferentes medios de comunicación, esto puede ir 
relacionado con la falta de capacitaciones o formación adecuada. Pese a esto consideran que es de suma importancia que estos temas se estudien desde primaria ya que es ahí en donde los educandos tienen los primeros contactos con las temáticas.

La información recolectada en este cuestionario muestra que la mayoría de participantes tienen una actitud positiva tanto hacia la estadística como a la probabilidad.

Una vez recopilada la información del cuestionario en los dos momentos citados, se realizó una comparación entre estos, con la finalidad de apreciar si la actitud de los docentes cambió después de la capacitación. Se aclara que al no ser una muestra aleatoria con la que se trabaja, las diferencias que se presentan no se puede afirmar si son significativas o no, solamente se pretende describir los cambios que se observaron en los promedios, porcentajes y desviaciones respectivas; el estudio es de tipo exploratorio.

\subsection{Comparación entre cuestionario inicial y final}

\subsubsection{Comparación de proposiciones negativas}

La información que se obtiene en los ítems iniciales y finales es muy similar. Aunque si se nota que la actitud en el cuestionario final es más positiva hacia las disciplinas en estudio. En el cuestionario inicial una parte de los participantes mostraba su indiferencia ante la afirmación la Estadística no sirve para nada, en el final la totalidad de participantes están en desacuerdo con esta frase. Respecto a si se debe o no enseñar Estadística y Probabilidad en la escuela, en el cuestionario inicial 5,88\% de los participantes considera que estas disciplinas no se deberían enseñar y en el final el 100\% de participantes están de acuerdo o muy de acuerdo con su enseñanza desde la educación primaria.

La comprensión de la información estadística que aparece en los medios de comunicación representa una dificultad para 37\% de los participantes (cuestionario inicial) este porcentaje disminuye a $30 \%$ en el cuestionario final. Situación similar ocurre en los ítems evito leer información estadística que aparece en la prensa y revistas y me siento intimidado ante datos estadísticos.

Al final del proceso de capacitación, una parte considerable de los docentes reconocen la utilidad que tienen las temáticas fuera de la escuela, siendo estos los ítems donde se demuestra el cambio de actitud mayor, ya que en el cuestionario inicial 70,59\% coinciden en utilizar poco la Probabilidad y $68,75 \%$ la Estadística y en el cuestionario final estos porcentajes disminuyen a $24 \%$ y $29 \%$ respectivamente. 
A continuación se realiza la comparación entre los cuestionarios respecto a los ítems positivos.

\subsubsection{Comparación de proposiciones positivas}

Al comparar los ítems positivos del cuestionario inicial y final, se notó que no hay cambios importantes en las opiniones de los enunciados propuestos y continúan pensando de manera similar.

Respecto al ítem Se debería eliminar la Estadística del currículo de primaria, este no presenta cambio pues concuerdan, en el inicial un $96 \%$ y en el final un $100 \%$, en que no se debería de eliminar el contenido del currículo escolar.

En cuanto a los enunciados sobre facilidad con la que perciben las temáticas, se notó una pequeña mejoría en el cuestionario final. Respecto al nivel de agrado, en el cuestionario final, los participantes opinan que su gusto por enseñar es un poco mayor en estadística que en probabilidad, situación similar ocurrió en el inicial. Por otra parte, en el inicial, un cierto número de docentes les resulta difícil la probabilidad o la estadística, aunque esta última es considerada más fácil por un mayor número de docentes; sin embargo, comparando los dos cuestionarios, para ambas áreas un gran número de participantes mencionan que son difíciles. Por lo que continúan pensando lo mismo, antes y después de las capacitaciones realizadas.

En ambos cuestionarios concuerdan en que por medio de los métodos estadísticos y probabilísticos se pueden tomar decisiones más documentadas y que es interesante el mundo de la Estadística para conocer y entender los fenómenos que los rodean.

En general, los docentes consideran que las disciplinas son útiles tanto en áreas de ciencias, como en situaciones de la cotidianeidad, por lo que es de suma importancia que estos temas se estudien desde la primaria. Tanto el cuestionario inicial como el final demuestran una actitud positiva de los docentes hacia la estadística y probabilidad e indican que no hubo cambios importantes en las opiniones de los participantes luego de las capacitaciones.

\section{Conclusiones}

En términos generales las opiniones expresadas en el cuestionario del inicio y del final de proceso de capacitación no tuvieron diferencias muy marcadas, en algunos aspectos la percepción tuvo una leve mejoría. 
Los participantes, aunque, consideran que estas temáticas ayudan a resolver problemas de la cotidianidad, muchos dicen no utilizarlas fuera del salón de clase, esto puede deberse a que, una parte de ellos considera que son temáticas difíciles de entender y por ende tratan de evitar estudios donde se analicen datos estadísticos o se tomen decisiones basadas en azar.

La mayoría de los participantes consideran a la estadística y a la probabilidad como temáticas de interés para la sociedad, que deben ser impartidas desde primaria ya que estamos rodeados de datos estadísticos; sin embargo, no todos se sienten a gusto cuando imparten estos temas, esta situación puede ser consecuencia de que su formación profesional en la universidad no fue del todo satisfactoria y hasta ese momento no habían recibido capacitaciones al respecto, situación preocupante ya que desde 1995 se incluyeron estas temáticas en el currículo y la mayoría de los participantes, tenían más de cinco años de experiencia por lo que podrían haber desarrollado estos temas en sus aulas.

Por otra parte, se presenta una contradicción, en las respuestas dadas, expresan que se pueden modelar situaciones cotidianas por medio de la aplicación de la estadística y la probabilidad, pero están de acuerdo en que con estas disciplinas se puede manipular la realidad, lo que indica que no consideran tan fidedignas las aplicaciones de las temáticas estudiadas en situaciones reales.

Como conclusión general urge la necesidad de hacer una revisión de los planes de estudio en las universidades donde se forman docentes de I y II ciclo de la Educación General Básica en cuanto a los cursos relacionados con Matemáticas ya que parece que no están siendo suficientes para que los docentes se sientan preparados una vez que llegan a las aulas. Además, los entes capacitadores de nuestro país como lo son: Instituto Uladislao Gámez, MEP, Universidades públicas y COLyPRO deben aportar a la sociedad costarricense ayudando con programas de actualización y capacitación continuas para docentes en servicio; ya que si el docente se siente capacitado en el área a enseñar su actitud hacia la misma es más positiva y por lo tanto trasmite eso a sus alumnos.

\section{Referencias}

Alfaro Arce, Ana Lucía, Alpízar Vargas, Marianela, Morales López, Yuri, Ramírez Bogantes, Melvin y Salas Huertas, Oscar. (2013). La formación inicial y continua de docentes de Matemáticas en Costa Rica. Cuadernos de Investigación y formación en Educación Matemática, 8(Especial), 131-179. 
Alpízar Vargas, Marianela, Barrantes, Jose Pablo, Bolaños, Hellen, Céspedes, Michael, Delgado, Evelyn, Freer, Dylna, Padilla, Eric y Víquez, María Fernanda. (2012). Aspectos relevantes sobre la formación docente en I y II ciclos en los temas Probabilidad y Estadística. Revista EDUCARE, 16(2), 113-129. Recuperado de http://www.revistas.una.ac.cr/index.php/EDUCARE/article/view/3934

Aparicio Pereda, Ana Sofía y Bazán Guzmán, Jorge Luis. (2006). Actitud y rendimiento en estadística en profesores peruanos. Acta Latinoamericana de Matemática Educativa, 19, 644-650. Recuperado de http://www.clame.org.mx/documentos/alme19.pdf

Barrantes, José Pablo, Bolaños, Hellen, Céspedes, Michael, Delgado, Evelyn, Freer, Dylna, Padilla, Eric y Víquez, María Fernanda. (2010). Estado actual de la enseñanza y aprendizaje de Probabilidad y Estadística, en I y II Ciclo, en la educación costarricense en las Direcciones regionales Educativas de Heredia y Pérez Zeledón. (Tesis para optar por el grado de Licenciatura en Enseñanza de la Matemática), Universidad Nacional, Heredia, Costa Rica.

Batanero Bernabeu, Carmen. (2001). Didáctica de la Estadística. Departamento de Didáctica de la Matemática de la Universidad de Granada. Recuperado de http://www.ugr.es/ batanero/pages/ARTICULOS/didacticaestadistica.pdf

Batanero Bernabeu, Carmen y Godino, Juan D. (2001). Análisis de Datos y su Didáctica. Departamento de Didáctica de la Matemática de la Universidad de Granada. Recuperado de http://www.ugr.es/ batanero/pages/ARTICULOS/Apuntes.pdf

Chaves Esquivel, Edwin. (2007). Inconsistencia entre los programas de estudio y la realidad de aula en la Enseñanza de la Estadística de Secundaria. Revista Actualidades Investigativas en Educación, 7(3), 1-35. Recuperado de http://revista.inie.ucr.ac.cr/autores/controlador/Article/accion/show/articulo/inconsistenci a-entre-los-programas-de-estudio-y-la-realidad-de-aula-en-la-ensenanza-de-laestadistica-de-secundaria.html

Estrada, Asunción. (2002). Análisis de las actitudes y conocimientos estadísticos elementales en la formación del profesorado. (Tesis para optar por el grado de Doctorado en Didáctica de la Matemática) Universidad Autónoma de Barcelona. Recuperado de http://www.ugr.es/ batanero/pages/librostesis.html

Jiménez, Liliana y Jiménez, José Rafael. (2005). Enseñar Probabilidad en primaria y secundaria ¿Para qué? y ¿Porqué? Revista Digital Matemática, Educación e Internet. 6(1). Recuperado de http://www.tec-digital.itcr.ac.cr/revistamatematica/contribucionesv6-n1-may2005/arti-aleat/index.html

Gattuso, Linda. (2006). Statistics and Mathematics. Is it possible to create fruitful links? En A. Rossman y B. Chance (Eds.), Proceedings of the Seventh International Conference on Teaching Statistics. CD ROM. Salvador (Bahia), Brazil: International Association for Statistical Education and International Statistical Institute.

Hernández Sampieri, Roberto, Fernández Collado, Carlos y Baptista Lucio, Pilar. (2006). Metodología de la Investigación (4a.ed.). México: McGraw-Hill Interamericana.

Holmes, Peter. (1980). Teaching statistics 11-16. Sloug: Foulsham Educational. 